\title{
Composition operators on a functional Hilbert space
}

\author{
R.K. Singh and S.D. Sharma
}

Let $T$ be a mapping from a set $X$ into itself and let $H(X)$ be a functional Hilbert space on the set $X$. Then the composition operator $C_{T}$ on $H(X)$ induced by $T$ is a bounded linear transformation from $H(X)$ into itself defined by $C_{T} f=f \circ T$. In this paper composition operators are characterized in the case when $H(X)=H^{2}\left(\pi^{+}\right)$in terms of the behaviour of the inducing functions in the vicinity of the point at infinity. An estimate for the lower bound of $\left\|C_{T}\right\|$ is given. Also the invertibility of $C_{T}$ is characterized in terms of the invertibility of $T$.

\section{Introduction and preliminaries}

Let $H(X)$ denote a functional Hilbert space on a set $X$, and let $T$ be a mapping from $X$ into itself. Then the composition mapping $C_{T}$, defined as

$$
C_{T} f=f \circ T
$$

maps $H(X)$ into the vector space of all complex-valued functions on $X$. This mapping $C_{T}$ is a linear transformation. If for every $f$ in $H(X)$, $C_{T} f$ is also in $H(X)$, then by the closed graph theorem $C_{T}$ is a bounded linear transformation on $H(X)$. The Banach algebra of all bounded linear transformations from $H(X)$ into itself is denoted by $B(H(X))$. If

Received 23 March 1979. 
$C_{T} \in B(H(X))$, we call it a composition operator on $H(X)$ induced by $T$. Some of the pertinent questions about these operators are: for which $T$ is the mapping $C_{T} \in B(H(X))$; when is an element $A \in B(H(X))$ a composition operator; if $C_{T} \in B(H(X))$, when is it invertible, compact, Fredholm, or normal? The answers of most of these questions are given by Nordgren [5] and Caughran and Schwartz [1]. If $H(X)$ is taken to be a nice well known functional Hilbert space, then some of the results obtained turn out to be very interesting. For example, if $H(X)=H^{2}(D)$, the classical Hardy space of the unit disc $D$, then every analytic mapping from $D$ into itself induces a composition operator [6].

In this note we are interested in the case when $H(X)$ is equal to $H^{2}\left(\pi^{+}\right)$, the Hilbert space of all those functions $f$ analytic on the upper half-plane $\pi^{+}$for which

$$
\sup _{y>0}\left\{\int_{-\infty}^{\infty}|f(x+i y)|^{2} d x\right\}<\infty .
$$

A characterization of all analytic mappings $T$ from $\pi^{+}$into itself for which $C_{T}$ is an operator on $H^{2}\left(\pi^{+}\right)$is reported in this paper in terms of the behaviour of $T$ in the neighbourhood of the point at infinity. An estimate of the norm of $C_{T}$ is given and the invertibility of $C_{T}$ is characterized.

The symbols $P$ and $\tilde{P}$ will stand for the Poisson integrals in the disc and in the upper half-plane respectively. The linear fractional transformation $L(z)=i(1+z) /(1-z)$ maps $D$ onto $\pi^{+}$and the unit circle onto the real line with $L^{-1}$ defined as $L^{-1}(w)=(w-i) /(w+i)$. The linear transformation $Q$ on $L^{2}(m)$, where $m$ is the normalized Lebesgue measure on the unit circle, defined by

$$
(Q f)(x)=(1 / \sqrt{\pi})\left(f \circ L^{-1}\right)(x) /(x+i),
$$

is a well established isometric isomorphism of $L^{2}(m)$ onto $L^{2}(\mu)$, where $\mu$ is the Lebesgue measure on the real line [4]. The set of all analytic functions $T: \pi^{+} \rightarrow \pi^{+}$such that the only singularity that $T$ can have is a pole at $\infty$ will be denoted by $A\left(\pi^{+}\right)$. 


\section{Boundedness of composition operators}

If $t$ is an analytic mapping from the unit disc $D$ into itself, then it is shown by Schwartz [6] that $C_{t}$ is a bounded operator on $H^{2}(D)$. But this is not true in the case of analytic mappings on $\pi^{+}$, as is shown later in an example in this section. In the following theorem a necessary and sufficient condition for an analytic mapping to induce a composition operator on $\mathrm{H}^{2}\left(\pi^{+}\right)$is given.

THEOREM 2.1. Let $T \in A\left(\pi^{+}\right)$. Then $C_{T}$ is a bounded operator on $H^{2}\left(\pi^{+}\right)$if and only if the point at infinity is a pole of $T$.

Proof. We first suppose that the point at infinity is a pole of $T$. Since $T$ is analytic in a neighbourhood of $\infty$, the function $t=L^{-1} \circ T \circ L$ is analytic in a neighbourhood of $I$, and $t(1)=1$. So by Corollary 2 of $[8], C_{T}$ belongs to $B\left(H^{2}\left(\pi^{+}\right)\right)$.

For necessity we suppose that $C_{T}$ is a bounded operator on $H^{2}\left(\pi^{+}\right)$. Then $f \circ T \in H^{2}\left(\pi^{+}\right)$for every $f \in H^{2}\left(\pi^{+}\right)$. Hence by Corollary 2 of [2, p. 191], $f(T(\omega))$ tends to zero as $w$ tends to infinity within each half-plane im $\omega \geq \delta>0$, where im $w$ stands for the imaginary part of $w$. Since the function $1 /(i+w)$ belongs to $H^{2}\left(\pi^{+}\right)$, it follows that $1 /(i+T(w))$ tends to zero as $w$ tends to infinity, which in turn implies that $T(w)$ tends to infinity as $w$ tends to infinity. This shows that the point at infinity is a pole of $T$.

COROLLARY 2.2. Let $T \in A\left(\pi^{+}\right)$. If $C_{T}$ is a bounded operator on $H^{2}\left(\pi^{+}\right)$, then $M_{B}$ belongs to $B\left(H^{2}(D)\right)$, where $M_{B}$ is the multiplication operator on $H^{2}(D)$ induced by $B(z)=(1-t(z)) /(1-z)$, and $t=L^{-1} \circ T \circ L$.

Proof. This follows trivially from Theorem 2.1.

EXAMPLES. (1) Let $T(w)=\alpha w+w_{0}$, where $a$ is a non-zero positive real number and $w_{0} \in \pi^{+}$. Then $T$ induces a composition operator on 
$H^{2}\left(\pi^{+}\right)$

(2) Let

$$
T(w)=i\left((w+i)^{n+1}+w(w-i)^{n}\right) /\left((w+i)^{n+1}-w(w-i)^{n}\right),
$$

where $n$ is a positive integer. Since the mapping

$$
t(z)=\left(L^{-1} \circ T \circ L\right)(z)=\frac{1}{2}\left(z^{n}+z^{n+1}\right)
$$

maps $D$ into itself, $T$ maps $\pi^{+}$into $\pi^{+}$. Also the point at infinity is a pole of $T$. Hence by Theorem 2.1, $C_{T} \in B\left(H^{2}\left(\pi^{+}\right)\right)$.

(3) Let $T(w)=(a w+b) /(c w+d)$, where $a, b, c, d \in R, a d-b c>0$ and $c \neq 0$. Then $T$ maps $\pi^{+}$onto itself, and by Theorem 2.1 it does not define a composition operator.

In [9] it is proved that if $t$ is an inner function from $D$ into itself, then $C_{T} \in B\left(H^{2}\left(\pi^{+}\right)\right)$implies that $t_{*}(1)=1$, where $T=L \circ t \circ L^{-1}$ and $t_{*}$ denotes the non-tangential limit of $t$. We prove this result in the following theorem for any analytic $t$, not necessarily an inner function.

THEOREM 2.3. Let $t$ be an analytic function from $D$ into itself and let $T=L \circ t \circ L^{-1}$. Then $C_{T} \in B\left(H^{2}\left(\pi^{+}\right)\right)$implies that $t_{*}(1)=1$.

Proof. Since $T$ is analytic and $C_{T}$ is a bounded operator on $H^{2}\left(\pi^{+}\right), \quad|T(w)| \rightarrow \infty$ as $|w| \rightarrow \infty$ within each half-plane im $w \geq \delta>0$, as is established in the proof of the necessary part of Theorem 2.1. Hence $t_{*}(1)=1$.

The converse of this theorem is not true as is obvious from the following example.

EXAMPLE. Let $t(z)=1-(1-z)^{\frac{1}{2}}$. Then $t$ induces a Hilbert-Schmidt composition operator on $H^{2}(D)$ [7]. Clearly $t_{*}(1)=1$ and

$$
T(\omega)=\left(L \circ t \circ L^{-1}\right)=(2(i \omega-1))^{\frac{1}{2}}-i .
$$


But $C_{T}$ is not bounded. This is because the function $f(w)=1 /(w+i)$ is a member of $H^{2}\left(\pi^{+}\right)$, but the function

$$
(f \circ T)(w)=I / \sqrt{2(i w-1)}
$$

is not in $H^{2}\left(\pi^{+}\right)$.

Let $N$ denote the set of all non-negative integers. For $n \in N$, define $S_{n}$ on $\pi^{+}$as

$$
S_{n}(w)=\left((w-i)^{n}\right) /\left(\sqrt{\pi}(w+i)^{n+1}\right)
$$

Then it is well known that the family $\left\{S_{n}: n \in N\right\}$ is an orthonormal basis for $H^{2}\left(\pi^{+}\right)$[4].

If $\alpha \in \pi^{+}$, then the reproducing kernel $k_{\alpha}$ for $H^{2}\left(\pi^{+}\right)$is defined by the equation

$$
\left\langle f, k_{\alpha}\right\rangle=f(\alpha)
$$

for all $f \in H^{2}\left(\pi^{+}\right)$. Using the above orthonormal basis it can be shown that

$$
k_{\alpha}(w)=\sum_{n=0}^{\infty}\left(\left((w-i)^{n}\right) /\left(\sqrt{\pi}(w+i)^{n+1}\right)\right)\left(\overline{\left((\alpha-i)^{n}\right) /\left(\sqrt{\pi}(\alpha+i)^{n+1}\right)}\right)
$$

[3, Problem 30].

A simple computation gives

$$
k_{\alpha}(w)=i / 2 \pi(w-\bar{\alpha})
$$

for every $w \in \pi^{+}$. Furthermore, the norm of $k_{\alpha}$ is given by

$$
\begin{aligned}
\left\|k_{\alpha}\right\|^{2} & =\left\langle k_{\alpha}, k_{\alpha}\right\rangle \\
& =k_{\alpha}(\alpha) \\
& =1 /(4 \pi \text { im } \alpha) .
\end{aligned}
$$

If $C_{T}$ is a composition operator, then the set of kernel functions is invariant under $C_{T}^{*}$, and in fact $C_{T}^{* k} k_{\alpha}=k_{T(\alpha)}$ [5]. This result is used 
in the following theorem to find a lower estimate for the norm of $C_{T}$.

THEOREM 2.4. If $C_{T}$ is a composition operator on $H^{2}\left(\pi^{+}\right)$, then

$$
\sup _{w \in \pi_{+}}\{(\text {im } w) /(\operatorname{im} T(w))\} \leq\left\|C_{T}\right\|^{2}
$$

Proof. For every $w \in \pi^{+}$, we have

$$
\begin{aligned}
((\operatorname{im} w) /(\operatorname{im} T(w))) & =\left\|k_{T(w)}\right\|^{2} /\left\|k_{w}\right\|^{2} \\
& =\left\|C_{T}^{* k} k_{w}\right\|^{2} /\left\|k_{w}\right\|^{2} \\
& \leq\left\|C_{T}^{*}\right\|^{2} \\
& =\left\|C_{T}\right\|^{2} .
\end{aligned}
$$

Since $w \in \pi^{+}$is arbitrary, it follows that

$$
\sup _{w \in \pi_{+}}\{(\operatorname{im} w) /(\operatorname{im} T(w))\} \leq\left\|C_{T}\right\|^{2} \text {. }
$$

\section{Invertibility of composition operators}

The invertibility of composition operators on $H^{2}(D)$ was studied by Schwartz [6]. He has shown that a composition operator is invertible if and only if it is induced by a conformal automorphism of the unit disc. We shall prove an analogous theorem on the invertibility of composition operators on $H^{2}\left(\pi^{+}\right)$by using an argument similar to that of Schwartz.

THEOREM 3.1. Suppose $T \in A\left(\pi^{+}\right)$and $C_{T} \in B\left(H^{2}\left(\pi^{+}\right)\right)$. Then $C_{T}$ is invertible if and only if $T$ is invertible.

Proof. Suppose $T$ is invertible. Since by Theorem 2.1 the point at infinity is a pole of $T$ and $T$ is invertible, it follows that the point at infinity is also a pole of $T^{-1}$, which shows that $C_{T^{-1}} \in B\left(H^{2}\left(\pi^{+}\right)\right)$. Clearly

$$
C_{T} C_{T}-1=C_{T^{-1}} C_{T}=I
$$

Therefore, 


$$
\left(C_{T}\right)^{-1}=C_{T^{-1}}
$$

Conversely, suppose $C_{T}$ is invertible. From Theorem 1 of [8] we have

$$
M_{B} C_{t}=P Q^{-1} \tilde{P}^{-1} C_{T} \tilde{P} Q P^{-1} \text {, }
$$

where $t=L^{-1} \circ T \circ L, C_{t}$ is the composition operator on $H^{2}(D)$ induced by $t$, and $M_{\beta}$ is the multiplication operator on $H^{2}(D)$ induced by $B(z)=(1-t(z)) /(1-z)$. Hence we can conclude that $M_{B} C_{t}$ is invertible. Since $M_{B}$ is subnormal and surjective, it is an invertible operator on $H^{2}(D)$. This is enough to conclude that $C_{t}$ is invertible on $H^{2}(D)$. From a theorem of [6] we get that $t$ is invertible, and consequently $T$ is invertible. This completes the proof of the theorem.

\section{References}

[1] James G. Caughran and Howard J. Schwartz, "Spectra of compact composition operators", Proc. Amer. Math. Soc. 51 (1975), 127-130.

[2] Peter L. Duren, Theory of $H^{p}$ spaces (Pure and Applied Mathematics, 38. Academic Press, New York and London, 1970).

[3] Paul R. Halmos, A Hilbert space problem book (Van Nostrand, Princeton, New Jersey; Toronto; London; 1967. Reprinted: Graduate Texts in Mathematics, 19. Springer-Verlag, New York, Heidelberg, Berlin, 1974).

[4] Kenneth Hoffman, Banach spaces of analytic functions (Prentice-Hall, Englewood Cliffs, New Jersey, 1962).

[5] Eric A. Nordgren, "Composition operators on Hilbert spaces", Hilbert space operators, 37-63 (Proc. Conf. Hilbert Space Operators, California State University, Long Beach, 1977. Lecture Notes in Mathematics, 693. Springer-Verlag, Berlin, Heidelberg, New York, 1978). 
[6] Howard J. Schwartz, "Composition operators on $\mathrm{H}^{\mathrm{p}}$ " (PhD thesis, University of Toledo, Ohio, 1969).

[7] J.H. Shapiro\&P.D. Taylor, "Compact, nuclear, and Hilbert-Schmidt composition operators on $H^{2}$ ", Indiana Univ. Math. J. 23 (1973), $471-496$.

[8] R.K. Singh, "A relation between composition operators on $H^{2}(D)$ and $H^{2}\left(\Pi^{+}\right) "$, Pure and App 2. Math. Sci. 1 (1974/75), no. 2, 1-5.

[9] R.K. Singh, "Inner functions and composition operators on a Hardy space", preprint.

Department of Mathematics,

University of Jammu,

jammu,

Tawi,

India. 